\title{
Specific inhibitors of urokinase plasminogen activator for treatment of cancers; In silico approach.
}

\author{
Benjamin A. Talbot (iD) $1 \quad a \quad b$ and Jerzy Jankun (iD) 2 \\ Coresponding author(s): \\ ${ }^{1}$ Benjamin.Talbot@ rockets.utoledo.edu ORCIID: https://orcid.org/0000-0002-0852-6432 \\ 2 JerzyJankun@utoledo.edu ORCID: https://orcid.org/0000-0003-2354-4046
}

\begin{abstract}
${ }^{a}$ Department of Urology, The University of Toledo - Health Science Campus, 3000 Arlington Ave., Toledo, OH 43614, USA, and ${ }^{b}$ M.D. Candidate, Class of 2023, The University of Toledo Heath Science Campus, 3000 Arlington Ave., Toledo, $\mathrm{OH} 43614$, USA.
\end{abstract}

Invasion, metastasis and angiogenesis are fundamental processes in the development of solid cancers. All these depend on the proteolysis where plasminogen activation system (PAS) is the prominent component. Plasmin of PAS, with a broad spectrum of proteins lysed, is secreted as a pro-enzyme and then activated by urokinase (uPA) or tissue plasminogen activator (tPA). Urokinase is in control of pericellular proteolysis while tPA mediates intravascular fibrinolysis. The most effective way to reduce excessive activity of plasmin in cancers is by inactivation of its activators (PAs). Inhibition of PAs reduces tumor size of cancers in vivo. However, for successful targeted anticancer therapy it is essential to find specific uPA inhibitors and to protect normal function of tPA. Unfortunately, most known inhibitors are unspecific, acting on both PAs. PAs are highly homologous enzymes with extreme similarities in their active sites so large numbers of chemicals need to be tested to find novel specific uPA inhibitors. Methods: As the availability of 3D protein structures determined experimentally by $\mathrm{X}$-ray crystallography is growing, computational methods are ever more used in targeted drug discovery. AutoDock Vina molecular docking, exploring binding of small molecules to target protein using a Monte Carlo technique and scoring function, is gaining popularity due to its excellent prediction accuracy. Results: Using AutoDock we have found many inhibitors of PA from our 3D database of $6170 \mathrm{com}$ pounds which bind in silico to $X$-ray structures of the specificity pocket (B187-B197, B212-B229) of both PAs preventing activation of plasminogen. However we were successful in identifying a few molecules which are specific for uPA. For example: both amiloride and chrysin bind to the specificity pocket of uPA but not to tPA. We have found that they bind to other parts of tPA, distant from the specificity pocket, thus preserving the tPA enzymatic activity while being effective toward uPA. Conclusion: In silico search yields specific inhibitors of uPA which, when verified for safety and efficiency by in vivo testing, could be used as novel therapeutics to limit metastasis and angiogenesis in anticancer therapy.

urokinase | tissue plasminogen activator | inhibitor | molecular modeling

D roteolysis is defined as the degradation of proteins by numerous proteolytic enzymes that plays a vital function in physiology and pathology of life forms. Proteolysis is closely controlled on the level of expression, activation, and inhibition in disease-free organisms. However, under-expression or over-expression of proteolytic activity is frequently observed in various diseases (1-4). One of the fundamental and common properties of malignant tumors is activation of proteolysis (5). In numerous malignancies, activated proteolytic enzymes are in control of local invasion, metastasis and angiogenesis (6-9). One of the members of the proteolytic family overexpressed in cancers is the plasminogen activation system (PAS), which include:

(i) Plasminogen: Secreted as a pro-enzyme that is cleaved by urokinase (uPA) or tissue plasminogen activator (tPA) and converted into its active form, plasmin. Plasmin digests a range of proteins and can activate other latent proteolytic enzymes $(5,6)$. Overactive plasmin is responsible for proteolysis in tissue remodeling, tumor invasion, development of distant metastasis, angiogenesis, and fibrinolysis $(5,9)$. 
(ii) Activators - uPA and tPA: These enzymes are weak proteases with high structural similarities. Both possess the primary function of activating plaminogen by proteolytic cleavage; uPA is in control of the activation of pericellular proteolysis while tPA mainly mediates intravascular fibrinolysis $(7,10,11)$.

(iii) The other members of the PAS such as the binding site of urokinase, called uPA receptor (UPAR), and inhibitors of plasminogen activators including PAI-1, and PAI-2, are not relevant to this paper $(8,12)$.

Since current therapeutic options for cancer patients are limited, novel tactics are needed $(9,13,14)$. The aberrant PAS activity in cancers can be explored to limit progression, metastasis, and angiogenesis in the potential therapeutic approach of PAS-targeted cancer therapies. In the activation cascade of the PAS, catalytic cleavage of plasminogen to plasmin is initiated by its activators uPA and tPA (6, $9,14,15)$. Thus, to control plasmin activity it is logical to normalize activity of plasminogen activators. In many cancers, abnormal plasmin activity is driven by elevated activity of uPA (16-18). uPA and tPA are highly homologous serine protease with extreme similarities in their active sites and with similar efficiency $(8,19-23)$.

Most known inhibitors of plasminogen activators are unspecific, acting not only on tPA and uPA but also to some extent on other serine proteases such as thrombin, trypsin and others (24-27). Thus, for successful targeted anticancer therapy, it is imperative to find specific uPA inhibitors, and at the same time protect normal function of other serine proteases of analogous structures, such as tPA. As the number of protein structures determined experimentally by X-ray crystallography and nuclear magnetic resonance spectroscopy is growing, computational methods are ever more used as a tool in targeted drug discovery (28-32). Among them, AutoDock Vina molecular docking (MD) methodology that predicts binding of small molecules to a target protein using a Monte Carlo sampling technique and scoring function, is gaining popularity due to its considerable improvement in prediction accuracy and docking time (33-38). By screening many molecules against uPA and tPA, predicted specific inhibitors of uPA should likely be identified.

\section{Materials and Methods}

\section{Chemicals identified as specific inhibitors of urokinase}

1. AMR, Amiloride. 3,5-diamino-6-chloro-N-(diaminomethylidene)-pyrazine-2-carboxamide.

2. CHY, Chrysin, 5,7-Dihydroxy-2-phenyl-4H-chromen-4-one.

3. 2H1, 7-(Propargyloxy)coumarin, 1-Benzopyran-2-one.

4. NHA, Nordihydroguaiaretic acid, 4-[4-(3,4-dihydroxyphenyl)2,3-dimethylbutyl]benzene-1,2-diol.

5. BAC, Baicalein, 5,6,7-trihydroxy-2-phenyl-4H-chromen-4one.

6. 3HO, AC1NDT8M, 3-(Hydroxymethyl)oxane-2,3,4,5-tetrol.

7. RBX, cis-Rubixanthin, 4-[(1E,3E,5E,7E,9E,11E,13E,15E, 17E,19E)-3,7,12,16,20,24-hexamethylpentacosa-1,3,5,7,9,11,13, 15,17,19,23-undecaenyl]-3,5,5-trimethylcyclohex-3-en-1-ol.

8. PSC, Psilocybine, [3-[2-(dimethylamino)ethyl]-1H-indol-4yl] dihydrogen phosphate.

9. HYC, L-Hyoscyamine, (8-methyl-8-azabicyclo[3.2.1]octan3-yl) 3-hydroxy-2-phenylpropanoate, acute toxic.

10. MTL, Meteloidine, [(6R,7S)-6,7-dihydroxy-8-methyl-8azabicyclo[3.2.1]octan-3-yl] (E)-2-methylbut-2-enoate.

11. CHE, CHEMBL195, [(1R,5R)-8-methyl-8-azabicyclo[3.2.1] -octan-3-yl] 3-hydroxy-2-phenylpropanoate.
12. AC1, AC1OFCHR, (2R,3S,4R,5R,6R)-6-methyloxane2,3,4,5-tetrol.

13. AGL, Andrographolide. (3E,4S)-3-[2-[(1R,4aS,5R,6R,8aS)6-hydroxy-5-(hydroxymethyl)-5, 8a-dimethyl-2-methylidene-3,4,4a,6,7,8-hexahydro-1H-naphthalen-1-yl]ethylidene]-4-hydroxyoxolan2-one.

14. HEM, CHEMBL69152, (2R,3R,4R,5R)-2-methylpiperidine3,4,5-triol.

\section{Acquisition of target enzyme}

The three-dimensional structures of human urokinase (4FUC, 1F5L) $(39,40)$ and human tPA (1A5H, 1BDA) $(41,42)$ were downloaded from the RCSB PDB database (43). In all target enzymes small molecules and water were removed using OpenBabel 2.4.1 (44) and were converted into PDBQT file format using PyRx (45).

\section{Ligand source}

Chemical structures of potential plasminogen activator inhibitors were obtained from our own internal databases created manually or extracted from PDB protein structures. Additionally, once the preliminary screening of our own databases was complete, the online PubChem chemistry database of the National Institutes of Health (46) was searched for compounds similar in structure to potential inhibitors identified from our databases, and the 3D structures of these similar compounds were downloaded in SDF format. Overall, 6,170 ligands were selected for screening.

\section{Ligand preparation}

When necessary, the two dimensional (2D) chemical structures of ligands were converted to three dimensional (3D) SDF structures using the "Baloon" (46) plugin for BioVia Draw (47), 3D structures of different formats (MOL and SDF), were converted to .pdbqt format using OpenBabel 2.4.1 functionality within PyRx $(44,45)$ in preparation for docking.

\section{Molecular docking}

PyRx's AutoDoc Vina program $(38,45)$ was utilized to dock ligands to the uPA and tPA enzymes. Whenever applicable both uncharged and partially charged ligands were screened at both the active sites and entire enzymes periphery to determine all possible locations of minimum binding energy. The entire enzyme structure of both UPA and tPA were selected to screen molecules. An "exhaustiveness" of 8 was selected within PyRx to utilize all 8 cores of the CPU $(38,45)$. A computer running the Windows 10 Professional, (10.0.18362 Build 18362) operating system with an Intel Core i7-8550u CPU, and 16.0 GB of physical memory was utilized for all functions of this project.

\section{Data Analysis}

An SDF file containing the 3D coordinates and predicted binding affinities of the docked ligands was exported from PyRx, then converted into a XYZ Cartesian coordinate file system by OpenBabel 2.4.1 (48) then opened with Microsoft Excel for quantitative analysis. Ligands with a difference of greater than $1.5 \mathrm{kcal} / \mathrm{mol}$ predicted affinity between binding to uPA versus tPA were further visualized. All ligands that bound within 10 Angstroms of the specificity site of uPA and greater than 15 Angstroms of the tPA specificity site were also further visualized.

In order to roughly determine the 3D straight line distance of docked molecules to the enzyme active site for both uPA and tPA, the key amino acids of the catalytic triad for each enzyme were 
identified, and the 3D molecular coordinates for the nitrogen in the first position of the imidazole ring of His57 was determined. Each molecule that was docked to an enzyme also had their 3D molecular coordinates for every atom extracted and then averaged to give $3 \mathrm{D}$ point roughly in the center of the molecule. The following formula was used to calculate distance:

$$
\text { distance }=\sqrt{\left(X_{H 57}-X_{i}\right)^{2}+\left(Y_{H 57}-Y_{i}\right)^{2}+\left(Z_{H 57}-Z_{i}\right)^{2}}
$$

where: $X, Y, Z$ are coordinates of His57 atom and center of inhibitor.

This provided a rough idea of the distance from the active site a molecule was predicted by AutoVina to dock, allowing for rapid identification of molecules that were docked within 10 Angstroms to His57 on uPA, but greater than 15 Angstroms from His57 of tPA.

\section{Visualization}

PyMol (48) was utilized for 3D visualization of the docking to compare the location of the molecule bound to uPA and tPA. LigPlot (49) was utilized to generate 2D schematics of selected ligandprotein binding.

\section{Results and Discussion}

Amiloride, known as a specific inhibitor of uPA, binds to the urokinase specificity pocket, but does not inhibit tPA, as it binds distant to its specificity site. To validate the Vina Autodock docking protocol we used this molecule as the positive control $(50,51,52)$.

As expected amiloride was docked in the predicted position within RMSD $<2.0$ Angstroms to uPA crystallographic structure (data not shown) (40) with strong calculated affinity $(-8.8 \mathrm{kcal} / \mathrm{mol})$. Calculations done for tPA and amiloride showed very weak affinity $(-4.3 \mathrm{kcal} / \mathrm{mol})$, and what is of great importance, the binding site of amiloride to tPA was distant from specificity pocket (26.9 Angstrom) further corroborating accuracy of established protocol.

It must be emphasized that in the course of the recurrent in silico simulations and generating of 3D structures, there are small variations in the affinity scores as well as 3D structure locations within protein structure. However, occasionally one or two simulations might be locked in a local minimum energy producing unusually different results from the rest of simulations. Therefore, each simulation presented in this paper was run ten times and only results having consistently small and negligible differences were accepted and presented (53-55). An ideal specific inhibitor could be one binding with high affinity to the specificity pocket of uPA, but in great distance from the specificity pocket of tPA, in a fashion that does not interfere with the binding of tPA to its substrates (plasminogen, PAI-1 or PAI-2).

Inhibiting serine proteases (including uPA and tPA) by blocking the specificity pocket is considered very effective since amino acids are major determinants the substrate specificity. Thus chemicals binding to uPA at the specificity pocket would less likely inhibit serine proteases family members such trypsin, chymotrypsin, elastase or others. However achieving inactivation of uPA but not IPA in that way remains elusive. This is due to the highly homologous nature of UPA and IPA especially in the specificity pocket where they are almost identical (40).

We decided to search the entirety of the uPA and tPA enzymes in hope that some ligands will have a higher predicted binding affinity for distant sites to the tPA specificity pocket, offering uPA inhibi- tion only. As expected, due to the homologous nature of uPA and tPA, the majority of screened compounds bound non-preferentially to both uPA and IPA within the specificity site.

Table 1. Calculated affinity of protein/inhibitors complexes and their distance from specificity pocket.

\begin{tabular}{ccccc}
\hline \hline Inhibitor & uPA $^{a}$ & distance $^{b}$ & tPA $^{a}$ & distance $^{c}$ \\
\hline AMR & -8.8 & 8.7 & -4.3 & 26.9 \\
CHY & -9.1 & 8.5 & -7.4 & 19.8 \\
2H1 & -6.1 & 7.8 & -6.2 & 19.3 \\
NHA & -7.1 & 9.8 & -7.4 & 26.5 \\
BAC & -8.2 & 8.9 & -7.4 & 26.4 \\
3HO & -5.5 & 6.0 & -4.9 & 22.7 \\
RBX & -7.1 & 12.1 & -7.1 & 22.3 \\
PSC & -6.1 & 9.5 & -6.0 & 23.7 \\
HYC & -6.4 & 10.1 & -5.9 & 19.8 \\
MTL & -6.7 & 6.0 & -6.5 & 23.3 \\
CHE & -6.2 & 8.9 & -6.1 & 19.4 \\
AC1 & -5.4 & 10.1 & -5.3 & 21.9 \\
AGL & -7.5 & 8.9 & -7.0 & 19.0 \\
HEM & -5.4 & 8.7 & -5.4 & 19.1 \\
\hline
\end{tabular}

a: affinity in $\mathrm{kcal} / \mathrm{mol}, \mathrm{b}$ : distance from $\mathrm{UPA}$ specificity pocket in Angstroms, $c$ : distance from tPA specificity pocket in Angstroms.
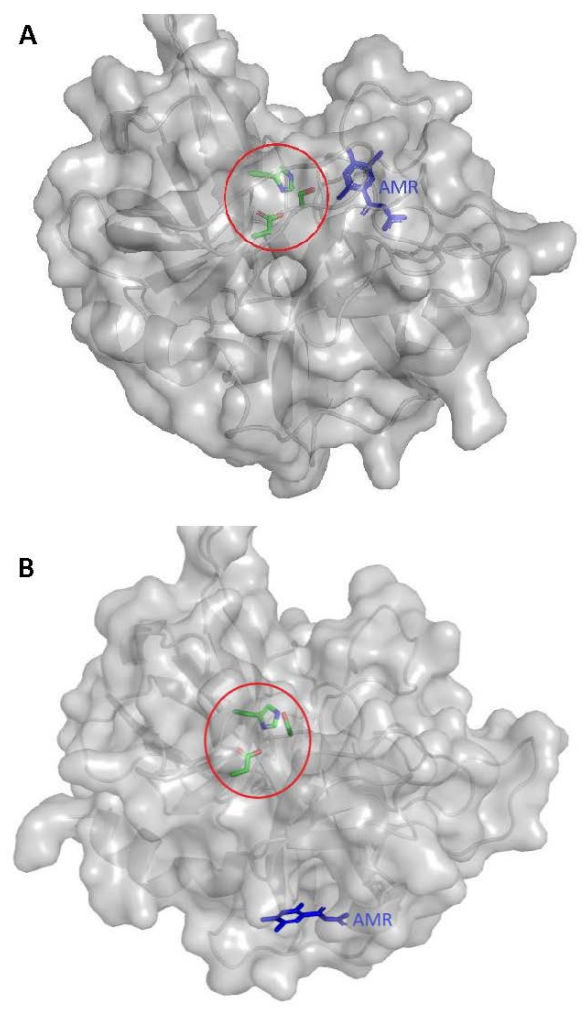

Fig. 1. A: uPA is shown as semitransparent surface, amiloride (dark blue) is docked in the specificity pocket, and amino acids of catalytic triad are shown as stick model, colored: green - carbon, blue - nitrogen, red-oxygen in the red circle. B: tPA amiloride is docked in a distant site from the specificity pocket. 

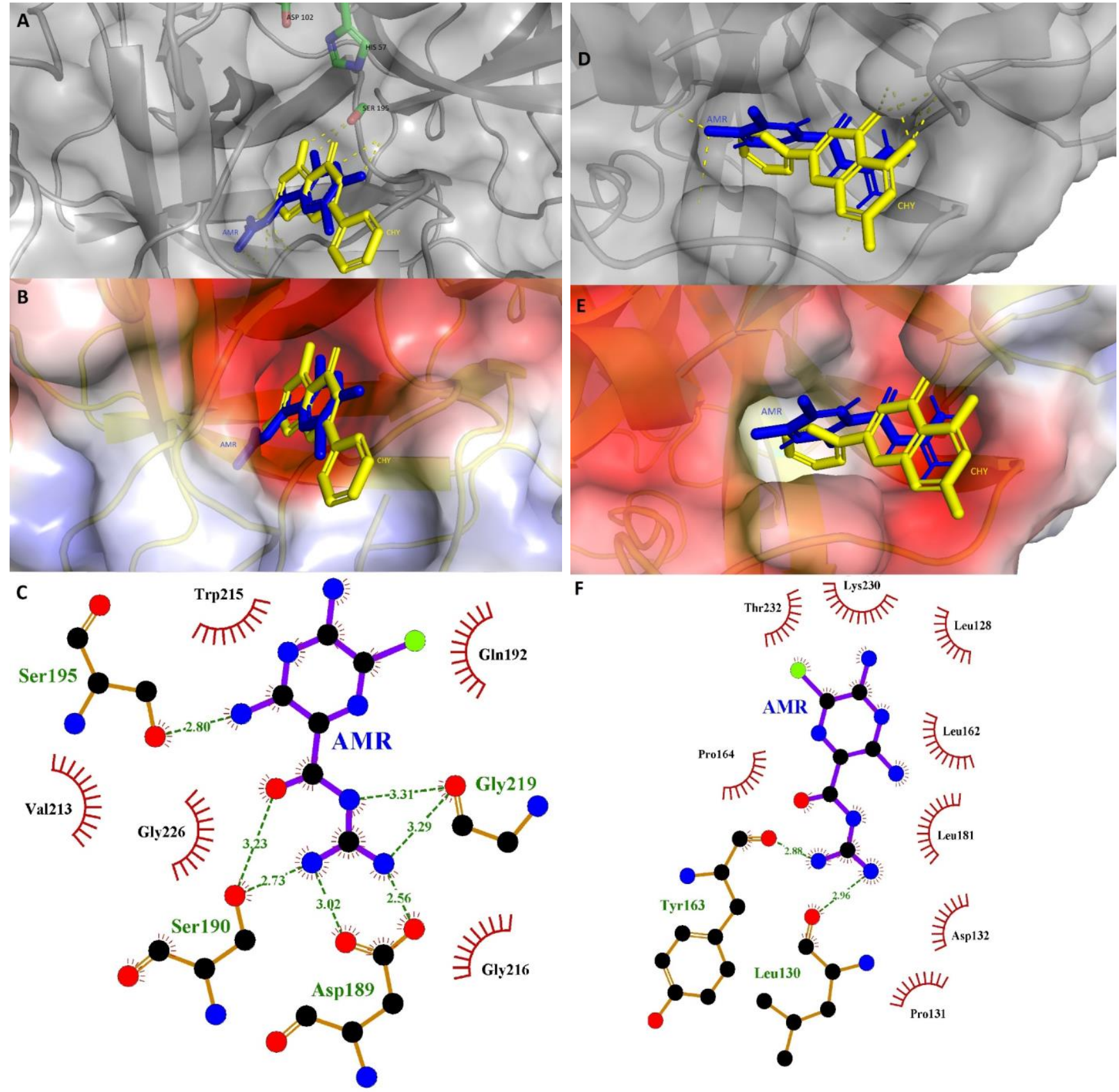

$\mathbf{F}$

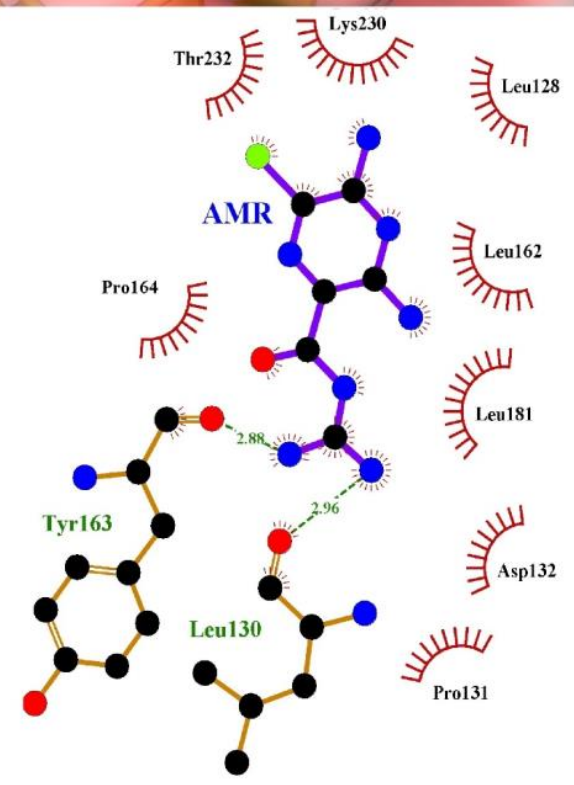

Fig. 2. A: uPA is shown as semitransparent surface, with amiloride (dark blue) and chrysin (yellow) docked in the specificity pocket, and amino acids of catalytic triad shown as stick model, colored: green - carbon, blue - nitrogen, red - oxygen. B: uPA surface colored by electrostatic potential: red negative $\left(-5 k_{b} T / e_{c}\right)$, blue - positive $\left(+5 k_{b} T / e_{c}\right)$, where: $k_{b}$ is Boltzmann's constant, $T$ is temperature in $K, e_{c}$ is the charge of an electron. Inhibitors of $u P A$ in the specificity pocket are shown as in A. C: Key amino acids of the specificity pocket interacting with amiloride by Ligand Plot. D: tPA is shown as semitransparent surface, with amiloride (dark blue) and chrysin (yellow) docked in the Leu128-Pro131 loop. E: tPA surface colored by electrostatic potential as in B. F: Key amino acid of Leu128-Pro131 pocket interacting with amiloride by Ligand Plot.

However, by comparing differences in predicted binding affinity as well as the predicted binding location of the screened compounds on both UPA and tPA, we can identify potential compounds that would act as specific inhibitors of uPA, while retaining enzymatic activity of tPA.
Of the 6170 molecules screened as potential specific inhibitors, fourteen molecules (including amiloride) were identified as binding close to the center of the urokinase specificity pocket, but far from the center of the tPA specificity site (Table 1). In addition to amiloride, are least three additional compounds (CHY, NHA, BAC) display greater affinity to uPA and are bound to tPA at a distance 
greater that 20 Angstroms from the tPA specificity pocket. Chrysin, a flavone found in honey, propolis, and passion flowers, has been investigated for anticancer activity and many possible pathways have been proposed (56).

Chen et al. reported decreased expression of uPA, and other enzymes at 24 and 48 hours when human melanoma cancer A375.S2 cells where treated with chrysin (57). Similarly Rondeau et al. found that NHA, a recognized inhibitor of lipoxygenase present in the creosote bush, (Larrea tridentata), reduces expression of uPA in LLC-PK1 cells (58).

It is interesting that only baicalein decreased the activity of the urokinase but not expression of uPA as it was reported in case of CHY and NHA (59). Specific inhibition of uPA, unknown until now can synergically increase anticancer activity of these compounds. Baicalein, a flavone, originally was isolated from the roots of Scutellaria baicalensis and Scutellaria lateriflora. This flavonoid has been shown to inhibit certain types of lipoxygenases and act on many other enzymes having curable effects on human diseases.

We were not able to find any references on inhibition of tPA in the literature for all of these three compounds promising that this approach could achieve significant selectivity for uPA inhibition.

1. Gharib SA, Manicone AM, and Parks WC (2018) Matrix metalloproteinases in emphysema. Matrix Biol 73:34-51.

2. Rabieian R, et al. (2018) Plasminogen Activator Inhibitor Type-1 as a Regulator of Fibrosis. J Cell Biochem 119(1):17-27.

3. Villalobos Acosta DMA, Chimal Vega B, Correa Basurto J, Fragoso Morales LG, and Rosales Hernandez MC (2018) Recent Advances by In Silico and In Vitro Studies of Amyloid-beta 1-42 Fibril Depicted a S-Shape Conformation. Int J Mol Sci 19(8).

4. Wilkinson DJ, Arques MDC, Huesa C, and Rowan AD (2019) Serine proteinases in the turnover of the cartilage extracellular matrix in the joint: implications for therapeutics. Br J Pharmacol 176(1):38-51.

5. Wyganowska-Swiatkowska M, Tarnowski M, Murtagh D, Skrzypczak-Jankun E, and Jankun $\mathrm{J}$ (2019) Proteolysis is the most fundamental property of malignancy and its inhibition may be used therapeutically (Review). Int $\mathrm{J}$ Mol Med 43(1):15-25.

6. Buckley BJ, Kelso MJ, Ali U, and Ranson M (2018) The Urokinase Plasminogen Activation System in Rheumatoid Arthritis: Pathophysiological Roles and Prospective Therapeutic Targets. Curr Drug Targets.

7. Jankun J, Khan OA, Mostafa HI, Sindhwani P, and Skrzypczak-Jankun E (2018) Can components of the plasminogen activation system predict the outcome of kidney transplants? Cent Eur J Immunol 43(2):222-230.

8. Jankun J and Skrzypczak-Jankun E (2009) Yin and yang of the plasminogen activator inhibitor. Pol Arch Med Wewn 119(6):410-417.

9. Madunic $J(2018)$ The Urokinase Plasminogen Activator System in Human Cancers: An Overview of Its Prognostic and Predictive Role. Thromb Haemost 118(12):2020-2036.

10. Jeanneret $V$ and Yepes $M$ (2016) The Plasminogen Activation System Promotes Dendritic Spine Recovery and Improvement in Neurological Function After an Ischemic Stroke. Transl Stroke Res.

11. Liu YX (2004) Plasminogen activator/plasminogen activator inhibitors in ovarian physiology. Front Biosci 9:3356-3373.

12. Shahrour $K$, Keck $R$, and Jankun $J$ (2015) Application of long-acting VLHL PAI-1 during sutureless partial nephrectomy in mice reduces bleeding. Biomed Res Int 2015:392862.

13. Li Y and Cozzi PJ (2007) Targeting uPA/uPAR in prostate cancer. Cancer Treat Rev 33(6):521-527.

14. McMahon BJ and Kwaan HC (2015) Components of the Plasminogen-Plasmin System as Biologic Markers for Cancer. Adv Exp Med Biol 867:145-156.

15. Rabbani SA and Xing RH (1998) Role of urokinase (UPA) and its receptor (UPAR) in invasion and metastasis of hormone-dependent malignancies. Int $J$ Oncol 12(4):911-920.

16. Akudugu J, Serafin A, and Bohm L (2015) Further evaluation of uPA and PAl-1 as biomarkers for prostatic diseases. J Cancer Res Clin Oncol 141(4):627-631.

17. LeBeau AM, et al. (2015) Imaging active urokinase plasminogen activator in prostate cancer. Cancer Res 75(7):1225-1235.

\section{Conclusion}

Therapy of malignancies by preventing invasion, metastasis and pathological angiogenesis should include downregulation of the variety of proteolytic enzymes. Examples include inhibitors of urokinase or metalloproteinase which could provide alternatives to existing anticancer therapies. However, at the same time we must preserve activity of physiologically important enzymes such as the tissue plasminogen activator. This enzyme is critical in normal coagulation but is highly homologous to urokinase, and therefore most of known inhibitors of uPA are also inactivators of tPA. This work provides evidence that it is possible to find highly specific inhibitors of urokinase while preserving activity of tPA for novel anticancer therapy.

\section{Conflict of interest}

Authors declare no conflict of interest.

\section{Authors' contributions}

BAT, JJ conceived and designed the experiments; BAT performed the calculations and formal analysis; JJ reviewed and revised the manuscript. Both authors wrote the manuscript, read and approved the final document.
18. Skovgaard D, Persson M, and Kjaer A (2017) Imaging of Prostate Cancer Using Urokinase-Type Plasminogen Activator Receptor PET. PET Clin 12(2):243-255.

19. Bode $W$ and Renatus $M$ (1997) Tissue-type plasminogen activator: variants and crystal/solution structures demarcate structural determinants of function.Curr Opin Struct Biol 7(6):865-872.

20. Lamba $D$, et al. (1996) The 2.3 A crystal structure of the catalytic domain of recombinant two-chain human tissue-type plasminogen activator. $J \mathrm{Mol}$ Biol 258(1):117-135.

21. Lin Z, et al. (2011) Structural basis for recognition of urokinase-type plasminogen activator by plasminogen activator inhibitor-1. J Biol Chem 286(9):7027-7032.

22. Spraggon G, et al. (1995) The crystal structure of the catalytic domain of human urokinase-type plasminogen activator. Structure 3(7):681-691.

23. Takehara S, et al. (2009) The 2.1-A crystal structure of native neuroserpin reveals unique structural elements that contribute to conformational instability. $\mathrm{J} \mathrm{Mol}$ Biol 388(1):11-20.

24. Bhongade BA, Gouripur VV, and Gadad AK (2005) 3D-QSAR CoMFA studies on trypsin-like serine protease inhibitors: a comparative selectivity analysis. Bioorg Med Chem 13(8):2773-2782.

25. Gladysz R, et al. (2015) Discovery and SAR of Novel and Selective Inhibitors of Urokinase Plasminogen Activator (UPA) with an Imidazo[1,2-a]pyridine Scaffold. J Med Chem 58(23):9238-9257.

26. Renatus M, Bode W, Huber R, Sturzebecher J, and Stubbs MT (1998) Structural and functional analyses of benzamidine-based inhibitors in complex with trypsin: implications for the inhibition of factor $\mathrm{Xa}$, tPA, and urokinase. $J$ Med Chem 41(27):5445-5456.

27. Zhang D, et al. (2017) Role of Vascular Endothelial Cells in Disseminated Intravascular Coagulation Induced by Seawater Immersion in a Rat Trauma Model. Biomed Res Int 2017:5147532.

28. Bello M (2018) Advances in Theoretical Studies on the Design of Single Boron Atom Compounds. Curr Pharm Des 24(29):3466-3475.

29. Gns HS, Gr S, Murahari M, and Krishnamurthy M (2019) An update on Drug Repurposing: Re-written saga of the drug's fate. Biomed Pharmacother 110:700-716.

30. Hoi PM, et al. (2015) Recent advances in structure-based drug design and virtual screening of VEGFR tyrosine kinase inhibitors. Methods 71:85-91.

31. Tamay-Cach F, et al. (2016) In Silico Studies Most Employed in the Discovery of New Antimicrobial Agents. Curr Med Chem 23(29):3360-3373.

32. Wang SH and $\mathrm{Yu} \mathbf{J}$ (2018) Structure-based design for binding peptides in anticancer therapy. Biomaterials 156:1-15.

33. Daniyan MO and Ojo OT (2019) In silico identification and evaluation of potential interaction of Azadirachta indica phytochemicals with Plasmodium falciparum heat shock protein 90. J Mol Graph Model 87:144-164.

34. Foroughi K, Khaksari M, and Shayannia A (2018) Molecular Docking Studies of Methamphetamine and Amphetamine-Related Derivatives as an Inhibitor against dopamine Receptor. Curr Comput Aided Drug Des. 
35. Kumar S, Khatik GL, and Mittal A (2018) In-silico Molecular Docking Study to Search New SGLT2 Inhibitor based on Dioxabicyclo[3.2.1] octane Scaffold. Curr Comput Aided Drug Des.

36. Sadati SM, Gheibi N, Ranjbar S, and Hashemzadeh MS (2019) Docking study of flavonoid derivatives as potent inhibitors of influenza H1N1 virus neuraminidase. Biomed Rep 10(1):33-38.

37. Sepehri B, Rezaei M, and Ghavami $R$ (2018) The in silico identification of poten anti-cancer agents by targeting the ATP binding site of the N-domain of HSP90. SAR QSAR Environ Res 29(7):551-565.

38. Trott $O$ and Olson $A J$ (2010) AutoDock Vina: improving the speed and accuracy of docking with a new scoring function, efficient optimization, and multithreading. J Comput Chem 31(2):455-461.

39. Kang YN, Stuckey, J.A., Nienaber, V., Giranda, V. (2012) Crystal Structure of the Urokinase. To be published.

40. Zeslawska E, et al. (2000) Crystals of the urokinase type plasminogen activator variant beta(c)-uPAin complex with small molecule inhibitors open the way towards structure-based drug design. Journal of molecular biology 301(2):465475.

41. Renatus M, et al. (1997) Structural mapping of the active site specificity determinants of human tissue-type plasminogen activator. Implications for the design of low molecular weight substrates and inhibitors. The Journal of biological chemistry 272(35):21713-21719.

42. Renatus M, et al. (1997) Lysine 156 promotes the anomalous proenzyme activity of tPA: X-ray crystal structure of single-chain human tPA. The EMBO journal 16(16):4797-4805.

43. Berman HM, et al. (2000) The Protein Data Bank. Nucleic Acids Research 28:235242.

44. O'Boyle NM, et al. (2011) Open Babel: An open chemical toolbox. Cheminformatics 3(33).

45. Dallakyan S and Olson AJ (2015) Small-Molecule Library Screening by Docking with PyRx. Chemical Biology: Methods and Protocols, eds Hempel JE, Williams CH, and Hong CC (Springer New York, New York, NY), pp 243-250.
46. Kim S CJ, Cheng T, Gindulyte A, He J, He S, Li Q, Shoemaker BA, Thiessen PA, Yu B, Zaslavsky L, Zhang J, Bolton EE. (2019) PubChem 2019 update: improved access to chemical data. Nucleic Acids Research.

47. BIOVIA DS (2018) BIOVIA Draw v18.1.

48. Schrödinger $L$ (2018) The PyMOL Molecular Graphics System, Version 2.0

49. Wallace AC LR, Thornton JM (1995) LIGPLOT: a program to generate schematic diagrams of protein-ligand interactions. Protein Engineering 8.

50. LB, Chi N, and Shi W (2015) Amiloride, a urokinase-type plasminogen activator receptor (UTPA) inhibitor, reduces proteinurea in podocytes. Genet Mol Res 14(3):9518-9529.

51. Vassalli JD and Belin $D$ (1987) Amiloride selectively inhibits the urokinase-type plasminogen activator. FEBS Lett 214(1):187-191.

52. Jankun J and Skrzypczak-Jankun E (2001) Binding site of amiloride to urokinase plasminogen activator depends on species. Int $J$ Mol Med 8(4):365-371.

53. Polinsky A, Goodman M, Williams KA, and Deber CM (1992) Minimum energy conformations of proline-containing helices. Biopolymers 32(4):399-406.

54. Sulimov VB, Kutov DC, and Sulimov AV (2018) Advances in Docking. Curr Med Chem.

55. Richardson JS, et al. (2018) Model validation: local diagnosis, correction and when to quit. Acta Crystallogr D Struct Biol 74(Pt 2):132-142.

56. Davatgaran-Taghipour $Y$, et al. (2017) Polyphenol nanoformulations for cancer therapy: experimental evidence and clinical perspective. Int $J$ Nanomedicine 12:2689-2702.

57. Chen HY, et al. (2019) Chrysin inhibit human melanoma A375.S2 cell migration and invasion via affecting MAPK signaling and NF-kappaB signaling pathway in vitro. Environ Toxicol 34(4):434-442.

58. Rondeau E, et al. (1990) Nordihydroguaiaretic acid inhibits urokinase synthesis by phorbol myristate acetate-stimulated LLC-PK1 cells. Biochim Biophys Acta 1055(2):165-172

59. Chiu YW, et al. (2011) Baicalein inhibits the migration and invasive properties of human hepatoma cells. Toxicol Appl Pharmacol 255(3):316-326. 